\title{
Layout and process optimisation: using computer-aided design (CAD) and simulation through an integrated systems design tool
}

\section{Luis M.S. Dias*, Guilherme A.B. Pereira, Pavel Vik and José A. Oliveira}

\author{
ALGORITMI Research Centre, \\ School of Engineering, \\ University of Minho, \\ Braga, Portugal \\ E-mail: luis.dias@algoritmi.uminho.pt \\ E-mail: guilherme.pereira@algoritmi.uminho.pt \\ E-mail:vikpavel@seznam.cz \\ E-mail: jose.oliveira@algoritmi.uminho.pt \\ ${ }^{*}$ Corresponding author
}

\begin{abstract}
The design of production and logistic systems is a process of managing both technical and organisational variants in order to identify the best solution for a given system. This paper discusses design issues of production systems that are applied to an internal logistic system in the automotive industry. As far as the production systems design (PSD) is concerned, three basic classes of software tools usually pertain: computer-aided design (CAD), process simulation, and information systems. However, these software tools have been used with low levels of integration. Vik et al. $(2010 \mathrm{~b}, 2010 \mathrm{c})$ proposed integrating these software resources in production systems and developed an advanced tool called integrated design of systems (IDS). The proposed IDS tool involves a wide set of functions for the most common tasks of PSD, from conceptualisation to implementation, including systems analysis (P-Q, cluster, and material flow analysis), automatic generation of simulation models, generation of alternatives for the layout of facilities and factories, material flows display, transportation system design, and iterative buffer size specification. The IDS approach takes advantage of simulation, CAD systems, and their integration. This paper will demonstrate the concept and functionalities of the proposed tool in a real industrial case study.
\end{abstract}

Keywords: integrated systems design; production systems planning and design; computer aided design; CAD; simulation; layout optimisation; process modelling; internal logistic; simulation automatic generation; system analysis; WITNESS; just-in-time; JIT.

Reference to this paper should be made as follows: Dias, L.M.S., Pereira, G.A.B., Vik, P. and Oliveira, J.A. (2014) 'Layout and process optimisation: using computer-aided design (CAD) and simulation through an integrated systems design tool', Int. J. Simulation and Process Modelling, Vol. 9, Nos. 1/2, pp.46-62.

Biographical notes: Luis M.S. Dias graduated in Computer Science and Systems Engineering at the University of Minho, Portugal. He holds an MSc degree in Computers Engineer. He holds a $\mathrm{PhD}$ degree in Production and Systems Engineering (Simulation) from the University of Minho. $\mathrm{He}$ is an Assistant Professor at University of Minho. His main research interests are modelling, simulation and optimisation.

Guilherme A.B. Pereira graduated in Industrial Engineering and Management in the University of Minho. He holds an MSc degree in Operational Research and $\mathrm{PhD}$ degree in Manufacturing and Mechanical Engineering from the University of Birmingham, UK. He is an Associate Professor at the University of Minho. His main research interests are modelling, simulation and operations research.

Pavel Vik studied at the Technical University of Liberec where he obtained his MSc in Manufacturing Systems. He holds a PhD degree in Industrial and Systems Engineering from the University of Minho. His main research interests are 3D animation and virtual reality in design of production systems and visualisation of computer simulation and automatic generating of simulation models.

José A. Oliveira studied Mechanical Engineering at the University of Porto. He holds a PhD in Production and Systems Engineering from the University of Minho. His main research interests are optimisation with heuristic methods in systems engineering. 
This article is a revised and expanded version of a paper entitled 'Integrated systems design in an automotive industry - using CAD and simulation in layout and process optimization' presented at MAS2012, Vienna, 2012.

\section{Introduction}

This paper deals with production systems design (PSD), particularly its improvement. It focuses on the design of systems and layouts based on material flows, re-layout processes, and the design of layouts influenced by different types of uncertainties.

Production systems must be developed or reorganised rapidly, efficiently, and with optimal configuration due to competition in the associated market worldwide and the rapid progress in many application areas, such as the building industry, manufacturing, information, communication, and all types of new technology. The time between customers' demands and production feedback must be as brief as possible. Organising a system should achieve high performance levels and cost-efficient layouts to reach optimum utilisation of resources, minimum transportation and logistics costs, maximum throughput rate, and minimum delivery times.

Due to the complexity of PSD, some specific software tools are frequently used that are classified as computer-aided design (CAD), process simulation, and information systems. While these tools help to improve the design process, they have been used with low levels of integration. The absence of data integration within these three classes of software tools and the lack of a systemic approach to PSD have caused work to be duplicated, time to be wasted, incoherencies, difficulties in project-team communication, and errors during the design phase. All setbacks lower the quality of possible solutions in the time available for design.

Therefore, the chief aim has been to conceptualise an integrated approach for system design and develop the correspondent application prototype to demonstrate the concept. Vik et al. (2010b, 2010c) developed and presented such a software tool, called integrated design of systems (IDS). According to the concept behind IDS, software tools are integrated into a unified system architecture solution with data coherence between the different tools to optimise the advantages of each class of tools. IDS was implemented on a database system, a CAD system for layout design, and a simulation tool. Simulation helps with dynamic systems analysis, while CAD helps with the static arrangement of a feasible implementation.

Any database system should provide an open structure to allow integration and data exchange between the simulation tool and the CAD system. A desirable database would hold everything concerning production system description, including all relevant data from different applications. The database should operate independently of the integrated application used, and its structure should provide for different levels of details. Furthermore, this solution should involve feedback between integrated tools.
Thus, changes in the production system configuration managed in one of the applications would affect the other application. This means that results from the simulations are used to improve $\mathrm{CAD}$ layout designs, and $\mathrm{CAD}$ layout data are inputted in new simulation experiments. This process should work iteratively in order to take advantage of the integrated approach.

Due to the abovementioned features of a desirable IDS, the software tools should involve a high automation level for the phases of PSD. Thus, the proposed solution would involve a wide set of developed functions for the most common PSD tasks from conceptualisation to implementation. Furthermore, IDS would support the automatic generation of alternatives for the layout of facilities and factories as well as generate automatic material flows display. Simulation models would then be automatically generated and configured, and simulation results would be loaded onto the database. The simulation model would provide full automatic background simulation.

Additionally, there is a set of functions for an iterative transportation system design (e.g., forklift and milk-run) and an iterative buffer-size specification. These functions would all be managed through user forms for input/output data control and results display.

Altogether, this approach would support a global system optimisation that considers all important system resources and system performance measures.

Given the benefits of such an approach, this paper will demonstrate this fully integrated PSD software tool. It begins by discussing the main issues related to the generic area of production systems (Section 2) and the main issues of production systems planning and design (Section 3). Section 4 discusses the main software applications used in production systems planning and design and emphasises the lack of integration. Section 5 briefly presents the approach followed by Vik et al. (2010b, 2010c) for developing IDS and the proposed aims associated with it. Section 6 addresses various issues of PSD when applied to an internal logistic system in the automotive industry. Section 7 introduces system data and discusses its inclusion in the IDS database. Sections 8 and 9 present the aforementioned integration of simulation and CAD within the IDS tool and address how these tools interact in order to get better solutions for the internal logistic system. Finally, Section 10 explains our conclusions.

\section{Production systems in brief}

A production system is organised as a set of elements that clarifies the relationships among these elements. System behaviour is based on the internal organisation and is influenced by the external environment. Relationships 